\title{
Solution of a linear pursuit-evasion game with integral constraints
}

G. I. Ibragimov ${ }^{1} \quad$ A. A. Azamov ${ }^{2} \quad$ M. Khakestari³

(Received 29 October 2010; revised 20 April 2011)

\begin{abstract}
A linear two player zero-sum pursuit-evasion differential game is considered. The control functions of players are subject to integral constraints. In the game, the first player, the Pursuer, tries to force the state of the system towards the origin, while the aim of the second player, the Evader, is the opposite. We construct the optimal strategies of the players when the control resource of the Pursuer is greater than that of the Evader. The case where the control resources of the Pursuer are less than or equal to that of the Evader is studied to prove the main theorem. For this case a new method for solving of the evasion problem is proposed. We assume that the instantaneous control employed by the Evader is known to the Pursuer. For construction, the strategy of the Evader information about the state of the system and the control resources of the players is used.
\end{abstract}

http://anziamj . austms.org.au/ojs/index.php/ANZIAMJ/article/view/3605 gives this article, (c) Austral. Mathematical Soc. 2011. Published June 3, 2011. ISSN 1446-8735. (Print two pages per sheet of paper.) Copies of this article must not be made otherwise available on the internet; instead link directly to this URL for this article. 


\section{Contents}

1 Introduction

E60

2 Main results

E63

3 An illustrative example

E71

4 Conclusion

E73

References

E73

\section{Introduction}

The study of linear two person zero-sum differential games was initiated by Isaacs [1]. Pontryagin [2], Berkovitz [3, 4], Krasovskii [5], Fleming [6, 7], Friedman [8], Elliott and Kalton [9], Petrosyan [10] and Hajek [11] developed the theory of differential games during 1960-1980.

Geometrical constraints are convenient for the mathematical study of control processes described by differential equations. Geometrical constraints are not the only way to model control processes. Moreover, they are not with reality. In real life, resources such as energy and fuel are restricted and hence we obtain integral constraints on control function.

Linear differential games with integral constraints on controls were examined in many works. Such games were slightly touched in the book of Issacs [1]. They were studied by Azimov [12,13] and Nikolskii [14] from the point of view of Pontryagin's first method. Later, different classes of linear differential games with integral constraints were investigated [14, 15, 16, 17, e.g.].

Ushakov [16] studied a linear differential game and showed that under some conditions the extremal strategy guarantees the termination of the pursuit at 
the instant of program absorption [16]. A differential game described by

$$
\dot{x}=A x+b u+c v
$$

where $u$ and $v$ are scalar control parameters, and $b$ and $c$ are $n$-vectors, was studied by Pshenichnii and Onopchuk [17]. Azamov and Samatov [18] examined a differential game with simple motions in depth. The optimal pursuit problem in the closed convex subset of $\mathbb{R}^{\mathfrak{n}}$ was studied by Ibragimov [19]. The work of Ibragimov [20] was devoted to the game described by an infinite system of differential equations with diagonal matrix. In this study the linear pursuit-evasion game for non-autonomous systems with integral constraints is considered. The objective of this article is to study a linear two person zero-sum pursuit-evasion differential game described by

$$
\dot{z}(\mathrm{t})=\mathrm{A}(\mathrm{t}) z+\mathrm{B}(\mathrm{t})(v-\mathrm{u}), \quad z(0)=z_{0} \neq 0, \quad z, z_{0} \in \mathbb{R}^{n},
$$

with the control parameters $u$ and $v$ satisfying integral constraints

$$
\int_{0}^{\infty}|u(t)|^{2} d t \leqslant \rho^{2}, \quad \int_{0}^{\infty}|v(t)|^{2} d t \leqslant \sigma^{2},
$$

where $A(t)$ and $B(t)$ are continuous $n \times n$ matrices, $t \geqslant 0$, the terminal set is $M=\{0\}$ and $\rho$ and $\sigma$ are given positive numbers.

Definition 1 A measurable function $u(t), u:[0, \infty) \rightarrow \mathbb{R}^{n}(v(t), v:$ $[0, \infty) \rightarrow \mathbb{R}^{n}$ ) subject to (2) is called an admissible control of the Pursuer (the Evader, respectively).

We denote by $\mathrm{U}_{\rho}$ (respectively $\mathrm{V}_{\sigma}$ ) as the set of all admissible controls of the Pursuer (Evader).

Definition 2 Pursuit is said to be completed in the game (1-2) if $z(\tau)=0$ at some $\tau \geqslant 0$.

The Pursuer tries to complete the game as early as possible, and the Evader has the opposite aim. 
Definition 3 A function $\mathrm{U}(\mathrm{t}, v), \mathrm{U}:[0, \infty) \times \mathbb{R}^{n} \rightarrow \mathbb{R}^{n}$, is called a strategy of the Pursuer if the system (1) has a unique absolutely continuous solution $z(\mathrm{t})=z\left(\mathrm{t}, z_{0}, \mathrm{U}, v(\cdot)\right), \mathrm{t} \geqslant 0$, at $\mathrm{u}=\mathrm{U}(\mathrm{t}, v)$ for every $v(\cdot) \in \mathrm{V}_{\sigma}$. A strategy of the Pursuer is called admissible if each control generated by this strategy is admissible.

Before the strategy of the Evader is defined, we extend the system (1-2) by introducing two new one dimensional state variables $p$ and $q$ by the equations

$$
\dot{p}=-|u|^{2}, \quad \dot{q}=-|v|^{2}, \quad p(0)=\rho^{2}, \quad q(0)=\sigma^{2} .
$$

Such an extension is a typical technique applied in studying games with integral constraints. If $\mathrm{t}$ is a current time, $\mathrm{u}(\cdot) \in \mathrm{U}_{\rho}$ and $v(\cdot) \in \mathrm{V}_{\sigma}$, then

$$
p(t)=\rho^{2}-\int_{0}^{t}|u(s)|^{2} d s, \quad q(t)=\sigma^{2}-\int_{0}^{t}|v(s)|^{2} d s .
$$

The functions $p(t)$ and $q(t)$ are called the control resources of the Pursuer and Evader, respectively. In the sequel, the four-tuple $(t, z, p, q)$ is referred as the state of the game.

Definition 4 A function $\mathrm{V}(\mathrm{t}, z, \mathrm{p}, \mathrm{q}), \mathrm{V}:[0, \infty) \times \mathbb{R}^{\mathrm{n}} \times \mathbb{R} \times \mathbb{R} \rightarrow \mathbb{R}^{\mathrm{n}}$, such that the systems (1) and (3) have a unique absolutely continuous solution $(z(\mathrm{t}), \mathrm{p}(\mathrm{t}), \mathrm{q}(\mathrm{t})), \mathrm{t} \geqslant 0$, at $\boldsymbol{v}=\mathrm{V}(\mathrm{t}, z, \mathrm{p}, \mathrm{q})$ for every $\mathrm{u}(\cdot) \in \mathrm{U}_{\rho}$ is called a strategy of the Evader. A strategy of the Evader is called admissible if each control generated by this strategy is admissible, that is, $\mathrm{V}(\mathrm{s}, z(\mathrm{~s}), \mathrm{p}(\mathrm{s}), \mathrm{q}(\mathrm{s}))$ is measurable and

$$
\int_{0}^{\infty}|\mathrm{V}(\mathrm{s}, z(\mathrm{~s}), \mathrm{p}(\mathrm{s}), \mathrm{q}(\mathrm{s}))|^{2} \mathrm{ds} \leqslant \sigma^{2}
$$

Definition 5 A finite number $\mathrm{T}$ is called the optimal pursuit time if the following two conditions hold:

1. there is a strategy $\mathrm{U}_{0}$ of the Pursuer such that for any $v(\cdot) \in \mathrm{V}_{\sigma}$ the equality $z\left(\mathrm{t}, z_{0}, \mathrm{U}_{0}, v(\cdot)\right)=0$ holds at some $\mathrm{t}=\tau \in[0, \mathrm{~T}]$ - in this case we say that the pursuit can be completed for the time $\mathrm{T}$; 
2. there is a strategy $\mathrm{V}_{0}$ of the Evader such that $z\left(\mathrm{t}, z_{0}, \mathrm{u}(\cdot), \mathrm{V}_{0}\right) \neq 0$ for any control $\mathrm{u}(\cdot) \in \mathrm{U}_{\rho}$ and $\mathrm{t} \in[0, \mathrm{~T})$-in this case we say that the evasion is possible on $[0, \mathrm{~T})$.

The strategies $\mathrm{U}_{0}$ and $\mathrm{V}_{0}$ are called the optimal strategies of the Pursuer and Evader, respectively.

The problem is to find the optimal pursuit time, and the optimal strategies of the Pursuer and the Evader in the game (1-2).

\section{Main results}

By Cauchy's formula, for $t \geqslant 0$, the solution of (1) has the form

$$
z(t)=\Psi(t)\left(z_{0}+\int_{0}^{t} \Psi^{-1}(s) B(s)(v(s)-u(s)) d s\right) .
$$

We assume that $\operatorname{det} B(t) \neq 0$, and $\rho>\sigma$. Let $\Psi(t)$ be a fundamental matrix solution of the homogeneous system $\dot{z}=A(t) z$, and $\Psi(0)=E$ be the unit matrix. Then the differential game defined by equation (1) is equivalent to the differential game described by

$$
\dot{z}_{1}=\mathrm{C}(\mathrm{t})(v(\mathrm{t})-\mathrm{u}(\mathrm{t})), \quad z_{1}(0)=z_{0},
$$

where $C(t)=\Psi^{-1}(t) B(t)$ is also continuous and nondegenerate. Solutions of equations (1) and (4) are connected by the equation $z_{1}(\mathrm{t})=\Psi^{-1}(\mathrm{t}) z(\mathrm{t})$. Let

$$
F(t)=\int_{0}^{t} C(s) C^{*}(s) d s, \quad t>0,
$$

where $C^{*}$ is transpose of the matrix $C$. This function $F(t)$ is symmetric and invertible. We consider an initial state $z_{0}$ such that

$$
z_{0} \mathrm{~F}^{-1}(\mathrm{t}) z_{0}=(\rho-\sigma)^{2}
$$


has a root $t=\theta$. The set of such initial states is not empty. For example, if $e$ is an arbitrary nonzero vector and $\vartheta$ is any positive number, then

$$
z_{0}=\lambda e, \quad \lambda=(\rho-\sigma)\left(e F^{-1}(\vartheta) e\right)^{-1 / 2}
$$

satisfies equation (6).

Lemma 6 The root of equation (6) is unique.

Proof: Indeed, differentiating $\mathrm{F}(\mathrm{t}) \mathrm{F}^{-1}(\mathrm{t})=\mathrm{E}$,

$$
F^{\prime}(t) F^{-1}(t)+F(t)\left(F^{-1}(t)\right)^{\prime}=0
$$

which implies

$$
\left(F^{-1}(t)\right)^{\prime}=-F^{-1}(t) F^{\prime}(t) F^{-1}(t)
$$

Then

$$
\begin{aligned}
z_{0}\left(\mathrm{~F}^{-1}(\mathrm{t})\right)^{\prime} z_{0} & =-\left\langle z_{0}, \mathrm{~F}^{-1}(\mathrm{t}) \mathrm{C}(\mathrm{t}) \mathrm{C}^{*}(\mathrm{t}) \mathrm{F}^{-1}(\mathrm{t}) z_{0}\right\rangle \\
& =-\left|\mathrm{C}^{*}(\mathrm{t}) \mathrm{F}^{-1}(\mathrm{t}) z_{0}\right|^{2}<0,
\end{aligned}
$$

where $\langle x, y\rangle$ is the inner product of the vectors $x$ and $y$. So the left hand side of equation (6) is a decreasing function of $t, t>0$. Hence the root $\theta$ of equation (6) is unique.

Theorem 7 If $\rho>\sigma$, then $\theta$, the root of equation (6), is the optimal pursuit time in the game (1-2).

Proof: We show that pursuit can be completed in the time $\theta$. We construct the strategy of the Pursuer as

$$
\mathrm{U}_{0}(\mathrm{t}, v)= \begin{cases}v+\mathrm{C}^{*}(\mathrm{t}) \mathrm{F}^{-1}(\theta) z_{0}, & 0 \leqslant \mathrm{t} \leqslant \theta \\ 0, & \mathrm{t}>\theta\end{cases}
$$


First, we check the admissibility of this strategy. Let $v(\cdot) \in \mathrm{V}_{\sigma}$ be an arbitrary control of the Evader, and let $u(t) \in U_{\rho}$ be defined by the equality $u(t)=U_{0}(t, v(t))$. Then according to the Minkowskii inequality, and the definition (5) of $\mathrm{F}(\mathrm{t})$ and (6),

$$
\begin{aligned}
\left(\int_{0}^{\infty}|\mathrm{u}(\mathrm{t})|^{2} \mathrm{dt}\right)^{1 / 2} & =\left(\int_{0}^{\theta}\left|v(\mathrm{t})+\mathrm{C}^{*}(\mathrm{t}) \mathrm{F}^{-1}(\theta) z_{0}\right|^{2} \mathrm{dt}\right)^{1 / 2} \\
& \leqslant\left(\int_{0}^{\theta}|v(\mathrm{t})|^{2} \mathrm{dt}\right)^{1 / 2}+\left(\int_{0}^{\theta}\left|\mathrm{C}^{*}(\mathrm{t}) \mathrm{F}^{-1}(\theta) z_{0}\right|^{2} \mathrm{dt}\right)^{1 / 2} \\
& \leqslant \sigma+\rho-\sigma=\rho .
\end{aligned}
$$

Now it can be shown that the strategy $(7)$ ensures the equality $z_{1}(\theta)=0$. Hence the pursuit can be completed for the time $\theta$.

Now we show that the evasion is possible on $[0, \theta)$. We construct a strategy $V_{0}$ for the Evader such that $z_{1}(t) \neq 0, t \in[0, \theta)$, for the trajectory $z_{1}(t)$ generated by $z_{0}, u(\cdot)$ and $V_{0}$, where $\mathfrak{u}(\cdot) \in \mathrm{U}_{\rho}$ is an arbitrary control function of the Pursuer.

We construct the Evader's strategy, $V_{0}$, in two steps.

1. The strategy $V_{0}$ is based on the fixed open-loop control function

$$
\mathbf{v}(t)=\frac{\sigma}{\rho-\sigma} C^{*}(t) F^{-1}(\theta) z_{0} \quad \text { if } p(t)>q(t) .
$$

This part of the strategy $V_{0}$ can be described briefly: the Evader uses the fixed control function $\mathbf{v}(t)$ while $p(t)>q(t)$. This inequality is true for the initial state $\left(0, z_{0}, \rho^{2}, \sigma^{2}\right)$.

2. Now we construct the strategy $V_{0}$ of the Evader for the position $\left(\mathrm{t}_{*}, z_{*}, \mathrm{p}_{*}, \mathrm{q}_{*}\right)$ such that $z_{*}=z_{1}\left(\mathrm{t}_{*}\right) \neq 0$ and $\mathrm{p}_{*}=\mathrm{p}\left(\mathrm{t}_{*}\right) \leqslant \mathrm{q}\left(\mathrm{t}_{*}\right)=\mathrm{q}_{*}$.

Let $T_{*}$ be any positive number. We partition the interval $\left[t_{*}, t_{*}+T_{*}\right]$ into subintervals by $t_{0}=t_{*}, t_{1}=t_{*}+h, \ldots, t_{i}=t_{*}+i h, \ldots$, 
$t_{n}=t_{*}+n h=t_{*}+T_{*}$. The number $h$ will be chosen later. We set

$$
\mathbf{v}(\mathrm{t})=0, \quad \mathrm{t}_{*} \leqslant \mathrm{t}<\mathrm{t}_{*}+\mathrm{h} .
$$

Let

$$
\begin{aligned}
& \mathrm{G}(\mathrm{k}+1)=\left(\int_{\mathrm{t}_{*}+\mathrm{kh}}^{\mathrm{t}_{*}+(\mathrm{k}+1) \mathrm{h}}\left|\mathrm{C}^{*}(\mathrm{t}) \mathrm{e}\right|^{2} \mathrm{dt}\right)^{1 / 2}, \quad e=\frac{z_{*}}{\left|z_{*}\right|}, \\
& \alpha_{\mathrm{k}}=\left(\int_{\mathrm{t}_{*}+(\mathrm{k}-1) \mathrm{h}}^{\mathrm{t}_{*}+\mathrm{kh}}|\mathrm{u}(\mathrm{t})|^{2} \mathrm{dt}\right)^{1 / 2}, \quad \mathrm{k}=1,2, \ldots
\end{aligned}
$$

We set, for $k=1,2, \ldots,(n-1)$,

$$
\mathbf{v}(\mathrm{t})=\alpha_{\mathrm{k}} \mathrm{G}^{-1}(\mathrm{k}+1) \mathrm{C}^{*}(\mathrm{t}) e, \quad \mathrm{t}_{*}+\mathrm{kh} \leqslant \mathrm{t}<\mathrm{t}_{*}+(k+1) \mathrm{h} .
$$

This constructs the strategy for the Evader.

We now show that the constructed strategy is admissible. Indeed, $\mathbf{v}(\mathbf{t})$ defined by (8) satisfies $\int_{0}^{\theta}|\mathbf{v}(\mathbf{t})|^{2} \mathbf{d t} \leqslant \sigma^{2}$. If $\mathbf{v}(\mathbf{t})$ is described by (9) and (10), then

$$
\begin{aligned}
\int_{t_{*}}^{t_{*}+T_{*}}|\mathbf{v}(t)|^{2} d t & =\sum_{k=0}^{n-1} \int_{t_{*}+k h}^{t_{*}+(k+1) h}|\mathbf{v}(t)|^{2} d t \\
& =\sum_{k=1}^{n-1} \int_{t_{*}+k h}^{t_{*}+(k+1) h} \alpha_{k}^{2} G^{-2}(k+1)\left|C^{*}(t) e\right|^{2} d t \\
& =\alpha_{1}^{2}+\cdots+\alpha_{n-1}^{2} \\
& =\int_{t_{*}}^{t_{*}+h}|u(t)|^{2} d t+\cdots+\int_{t_{*}+(n-2) h}^{t_{*}+(n-1) h}|\mathfrak{u}(t)|^{2} d t \\
& \leqslant \int_{t_{*}}^{t_{*}+T_{*}}|\mathfrak{u}(t)|^{2} d t \leqslant p_{*} \leqslant q_{*} .
\end{aligned}
$$

Therefore, the constructed strategy of the Evader is admissible.

We now consider the case $p(t)>q(t)$. We prove the following Lemma. 
Lemma 8 Let $\mathbf{v}(\mathrm{t})$ be defined by (8). Then $z_{1}(\mathrm{t}) \neq 0$ for any $\mathbf{u}(\cdot) \in \mathrm{U}_{\rho}$ while $\mathrm{p}(\mathrm{t})>\mathrm{q}(\mathrm{t}), 0 \leqslant \mathrm{t}<\theta$. Moreover, $z_{1}\left(\mathrm{t}_{*}\right) \neq 0$ at the first time $\mathrm{t}_{*} \in(0, \theta)$ where $\mathrm{p}\left(\mathrm{t}_{*}\right)=\mathrm{q}\left(\mathrm{t}_{*}\right)$.

Proof: Let us assume the contrary:

$$
z_{1}(\tau)=0, \quad p(\tau) \geqslant q(\tau)
$$

for some $\tau \in(0, \theta)$. Hence, $0=z_{1}(\tau)=z_{0}+\int_{0}^{\tau} C(t)(v(t)-u(t)) d t$. Then according to (8) we obtain

$$
\begin{aligned}
\int_{0}^{\tau} C(t) u(t) d t & =z_{0}+\int_{0}^{\tau} C(t) \mathbf{v}(t) d t \\
& =z_{0}+\int_{0}^{\tau} C(t) \frac{\sigma}{\rho-\sigma} C^{*}(t) F^{-1}(\theta) z_{0} d t \\
& =z_{0}+\frac{\sigma}{\rho-\sigma} F(\tau) F^{-1}(\theta) z_{0}
\end{aligned}
$$

Now we use the following [21].

Assertion 9 Let $\mathrm{C}(\mathrm{t}), 0 \leqslant \mathrm{t} \leqslant \mathrm{T}$, be continuous $\mathrm{n} \times \mathrm{n}$-matrix, and its determinant be not identically zero on $[0, \mathrm{~T}]$. Then among the measurable functions $\mathrm{u}(\cdot), \mathfrak{u}:[0, \mathrm{~T}] \rightarrow \mathbb{R}^{\mathrm{n}}$, satisfying the condition $\int_{0}^{\mathrm{T}} \mathrm{C}(\mathrm{s}) \mathrm{u}(\mathrm{s}) \mathrm{d} \mathrm{s}=\xi$, the control defined by the formula

$$
\mathfrak{u}(\mathrm{s})=\mathrm{C}^{*}(\mathrm{~s}) \mathrm{F}^{-1}(\mathrm{~T}) \xi \text {, a.e. on }[0, \mathrm{~T}] \text {, where } \mathrm{F}(\mathrm{T})=\int_{0}^{\mathrm{T}} \mathrm{C}(\mathrm{s}) \mathrm{C}^{*}(\mathrm{~s}) \mathrm{ds} \text {, }
$$

gives the minimum to the functional $\int_{0}^{\mathrm{T}}|\mathrm{u}(\mathrm{s})|^{2} \mathrm{ds}$.

If $\xi$ equals the right part of (12) and $\mathrm{T}=\tau$, then according to Assertion 9 the control

$$
u(t)=C^{*}(t) F^{-1}(\tau)\left(z_{0}+\frac{\sigma}{\rho-\sigma} F(\tau) F^{-1}(\theta) z_{0}\right), \quad 0 \leqslant t \leqslant \tau,
$$


gives the minimum to the functional $\int_{0}^{\tau}|u(s)|^{2} \mathrm{ds}$. As proved above the function $f(t)=z_{0} F^{-1}(t) z_{0}$ is decreasing. Hence, combining (6), (8) and (13), yields

$$
\begin{aligned}
\int_{0}^{\tau}|u(s)|^{2} \mathrm{ds}-\int_{0}^{\tau}|\mathbf{v}(s)|^{2} \mathrm{ds} & \geqslant z_{0} \mathrm{~F}^{-1}(\tau) z_{0}+\frac{2 \sigma}{\rho-\sigma} z_{0} \mathrm{~F}^{-1}(\theta) z_{0} \\
& >z_{0} \mathrm{~F}^{-1}(\theta) z_{0}+\frac{2 \sigma}{\rho-\sigma} z_{0} \mathrm{~F}^{-1}(\theta) z_{0} \\
& =(\rho-\sigma)^{2}+2 \rho \sigma-2 \sigma^{2}=\rho^{2}-\sigma^{2} .
\end{aligned}
$$

Hence,

$$
\rho^{2}-\int_{0}^{\tau}|u(s)|^{2} \mathrm{ds}<\sigma^{2}-\int_{0}^{\tau}|\mathbf{v}(s)|^{2} \mathrm{ds}
$$

which means $p(\tau)<q(\tau)$, which contradicts (11). So the proof of Lemma 8 is complete.

We show that the evasion is possible in the case $p\left(t_{*}\right) \leqslant q\left(t_{*}\right)$. We assume that at some time $t_{*}, 0 \leqslant t_{*}<\theta$, the equality $p\left(t_{*}\right)=q\left(t_{*}\right)$ occurred. At this time

$$
\int_{\mathrm{t}_{*}}^{\infty}|\mathrm{u}(\mathrm{s})|^{2} \mathrm{~d} s \leqslant \mathrm{p}_{*}, \quad \int_{\mathrm{t}_{*}}^{\infty}|v(s)|^{2} \mathrm{ds} \leqslant \mathrm{q}_{*}=\mathrm{p}_{*} .
$$

According to Lemma $8, z_{*}=z_{1}\left(t_{*}\right) \neq 0$. We need to show that evasion is possible on the interval $\left[t_{*}, \theta\right)$. It is a consequence of the following lemma where we should take $T_{*}=\theta-t_{*}$.

Lemma 10 If $\mathrm{p}\left(\mathrm{t}_{*}\right) \leqslant \mathrm{q}\left(\mathrm{t}_{*}\right), z_{1}\left(\mathrm{t}_{*}\right)=z_{*} \neq 0$, then evasion is possible on $\left[\mathrm{t}_{*}, \mathrm{t}_{*}+\mathrm{T}_{*}\right]$ from the initial point $\boldsymbol{z}_{*}$.

Proof: We use the second part of the strategy (8-9). For simplicity of calculations we take $t_{*}=0$. To show the inequality $z_{1}(t) \neq 0$, it is sufficient to establish that $\mathrm{d}(\mathrm{t})=\left\langle e, z_{1}(\mathrm{t})\right\rangle>0$. We have

$$
\mathrm{d}(\mathrm{t})=\left\langle e, z_{*}+\int_{0}^{t} \mathrm{C}(\mathrm{s}) \mathbf{v}(\mathrm{s}) \mathrm{ds}\right\rangle-\left\langle e, \int_{0}^{t} \mathrm{C}(\mathrm{s}) \mathrm{u}(\mathrm{s}) \mathrm{ds}\right\rangle
$$




$$
\begin{aligned}
= & \left\langle e, z_{*}+\sum_{k=1}^{m} \int_{(k-1) h}^{k h} C(s) \mathbf{v}(s) d s+\int_{m h}^{t} C(s) \mathbf{v}(s) d s\right\rangle \\
& -\left\langle e, \sum_{k=1}^{m} \int_{(k-1) h}^{k h} C(s) u(s) d s+\int_{m h}^{t} C(s) u(s) d s\right\rangle
\end{aligned}
$$

where $t \in(m h,(m+1) h], m+1 \leqslant n$. Since $\langle e, C v\rangle=\left\langle C^{*} e, v\right\rangle$ and by the definition of $\mathbf{v}, \int_{0}^{h} \mathrm{C}(\mathrm{s}) \mathbf{v}(\mathrm{s}) \mathrm{ds}=0$, then

$$
\begin{aligned}
\mathrm{d}(\mathrm{t})= & \left|z_{*}\right|+\sum_{k=2}^{\mathrm{m}} \int_{(k-1) \mathrm{h}}^{\mathrm{kh}}\left\langle\mathrm{C}^{*}(\mathrm{~s}) e, \mathrm{v}(\mathrm{s})\right\rangle \mathrm{d} s+\int_{\mathrm{mh}}^{\mathrm{t}}\left\langle\mathrm{C}^{*}(\mathrm{~s}) e, \mathbf{v}(\mathrm{s})\right\rangle \mathrm{d} s \\
& -\sum_{\mathrm{k}=1}^{\mathrm{m}} \int_{(\mathrm{k}-1) \mathrm{h}}^{\mathrm{kh}}\left\langle\mathrm{C}^{*}(\mathrm{~s}) e, \mathrm{u}(\mathrm{s})\right\rangle \mathrm{d} \mathrm{s}-\int_{\mathrm{mh}}^{\mathrm{t}}\left\langle\mathrm{C}^{*}(\mathrm{~s}) e, \mathrm{u}(\mathrm{s})\right\rangle \mathrm{d} s .
\end{aligned}
$$

For the constructed strategy of the Evader,

$$
\int_{k h}^{(k+1) h}\left\langle C^{*}(s) e, v(s)\right\rangle d s=\alpha_{k} G(k+1) .
$$

Also for any control of the Pursuer,

$$
\begin{aligned}
\int_{k h}^{(k+1) h}\left\langle C^{*}(s) e, u(s)\right\rangle d s & \leqslant \int_{k h}^{(k+1) h}\left|C^{*}(s) e\right||u(s)| d s \\
& \leqslant \sqrt{\int_{k h}^{(k+1) h}\left|C^{*}(s) e\right|^{2} d s} \sqrt{\int_{k h}^{(k+1) h}|u(s)|^{2} d s} \\
& \leqslant \alpha_{k+1} G(k+1) .
\end{aligned}
$$

According to (14) and (15) we obtain

$$
\begin{aligned}
d(t) & \geqslant\left|z_{*}\right|+\sum_{k=2}^{m} \alpha_{k-1} G(k)-\sum_{k=1}^{m+1} \alpha_{k} G(k) \\
& \geqslant\left|z_{*}\right|+\sum_{k=2}^{m} \alpha_{k-1}(G(k)-G(k-1))-\sqrt{p_{*}}(G(m)+G(m+1))
\end{aligned}
$$


since $\alpha_{k} \leqslant \sqrt{p_{*}}$. We now estimate

$$
\begin{aligned}
& \left|\sum_{k=1}^{m-1} \alpha_{k}(G(k+1)-G(k))\right| \\
\leqslant & \sum_{k=1}^{m-1} \alpha_{k}|G(k+1)-G(k)| \\
\leqslant & \left(\sum_{k=1}^{m-1} \alpha_{k}^{2}\right)^{1 / 2}\left(\sum_{k=1}^{m-1}|G(k+1)-G(k)|^{2}\right)^{1 / 2} .
\end{aligned}
$$

Here, we use the Cauchy-Schwartz inequality. We get,

$$
\sum_{k=1}^{m-1} \alpha_{k}^{2}=\sum_{k=1}^{m-1} \int_{(k-1) h}^{k h}|u(t)|^{2} d t=\int_{0}^{(m-1) h}|u(t)|^{2} d t \leqslant \int_{0}^{\infty}|u(t)|^{2} d t \leqslant p_{*} .
$$

Denote

$$
\omega_{f}(h)=\sup \left\{\left|f\left(t^{\prime}\right)-f\left(t^{\prime \prime}\right)\right|: t^{\prime}, t^{\prime \prime} \in\left[0, T_{*}\right],\left|t^{\prime}-t^{\prime \prime}\right| \leqslant h\right\} .
$$

Now verify that $\omega_{f}(h)$ has the following two properties: $\omega_{f}(h) \geqslant 0$; the function $f(t), 0 \leqslant t \leqslant T_{*}$, is uniformly continuous if and only if $\lim _{h \rightarrow 0} \omega_{f}(h)=0$. Taking $\mathrm{f}(\mathrm{t})=\left|\mathrm{C}^{*}(\mathrm{t}) e\right|^{2}$, yields

$$
\begin{aligned}
\sum_{k=1}^{m-1}|G(k+1)-G(k)|^{2} & \leqslant \sum_{k=1}^{m-1}\left|G^{2}(k+1)-G^{2}(k)\right| \\
& =\left.\sum_{k=1}^{m-1}\left|\int_{k h}^{(k+1) h}\right| C^{*}(s) e\right|^{2} d s-\int_{(k-1) h}^{k h}\left|C^{*}(s) e\right|^{2} d s \mid \\
& \leqslant\left.\sum_{k=1}^{m-1} \int_{(k-1) h}^{k h}|| C^{*}(s+h) e\right|^{2}-\left|C^{*}(s) e\right|^{2} \mid d s \\
& \leqslant \sum_{k=1}^{m-1} \omega_{f}(h) h \leqslant \omega_{f}(h) m h \leqslant T_{*} \omega_{f}(h)
\end{aligned}
$$


By combining (17), (18) and (19), we obtain

$$
\left|\sum_{k=1}^{m-1} \alpha_{k}(G(k+1)-G(k))\right| \leqslant \sqrt{p_{*} T_{*} \omega_{f}(h)} .
$$

We now estimate $\sqrt{p_{*}}(G(m)+G(m+1))$ in (16). Let $K=\max _{t \in\left[0, T_{*}\right]}\left|C^{*}(t) e\right|$. Then

$$
\sqrt{p_{*}}(G(m)+G(m+1)) \leqslant 2 K \rho \sqrt{h} .
$$

As the function $f(t)$ defined above is continuous it is uniformly continuous. So $\lim _{h \rightarrow 0} \omega_{f}(h)=0$. If we choose $h$ to satisfy

$$
\sqrt{p_{*} T_{*} \omega_{f}(h)}+2 K \sqrt{p_{*} h}<\left|z_{*}\right| / 2,
$$

then according to (16), (20) and (21)

$$
d(t) \geqslant\left|z_{*}\right|-\left(\sqrt{p_{*} T_{*} \omega_{f}(h)}+2 K \sqrt{p_{*} h}\right)>\frac{1}{2}\left|z_{*}\right| .
$$

Thus, the Evader can choose $h$ such that $d(t)>\frac{1}{2}\left|z_{*}\right|$. Consequently, evasion is possible in the game (1-2). This completes the proof of Lemma 10. Hence, the proof of Theorem 7 is complete.

\section{An illustrative example}

Here, we give an illustrative example that reduces to the system (4) and hence Theorem 7 applies. We consider a differential game with one Pursuer P and one Evader $\mathrm{E}$ whose motions are described by the equations

$$
\begin{array}{ll}
\dot{x}=y+u_{1}-v_{1}, & x(0)=x_{0}, \\
\dot{y}=-x+u_{2}-v_{2}, & y(0)=y_{0},
\end{array}
$$

where $x, y, x_{0}, y_{0}, \mathfrak{u}_{1}, \mathfrak{u}_{2}, v_{1}, \mathfrak{u}_{2} \in \mathbb{R}^{1}$. The control function $\mathfrak{u}(\cdot)$ (respectively, $v(\cdot))$ of the Pursuer $\mathrm{P}$ (Evader E) satisfies the inequalities (2). The 
game is regarded as ended when $x(t)=y(t)=0$ for some $t>0$. Using the representation

$$
\left[\begin{array}{c}
\dot{x} \\
\dot{y}
\end{array}\right]=\left[\begin{array}{cc}
0 & 1 \\
-1 & 0
\end{array}\right]\left[\begin{array}{l}
x \\
y
\end{array}\right]+\left[\begin{array}{l}
u_{1}-v_{1} \\
u_{2}-v_{2}
\end{array}\right],
$$

and vector $z=(x, y)$ equation (22) becomes

$$
\dot{z}=\mathrm{A} z+\mathrm{B}(\mathrm{u}-v), \quad z(0)=z_{0}=\left[\begin{array}{l}
x_{0} \\
y_{0}
\end{array}\right],
$$

where

$$
A=\left[\begin{array}{cc}
0 & 1 \\
-1 & 0
\end{array}\right], \quad B=\left[\begin{array}{ll}
1 & 0 \\
0 & 1
\end{array}\right]
$$

The fundamental matrix is defined by the formula

$$
X(t)=\left[\begin{array}{cc}
\cos t & \sin t \\
-\sin t & \cos t
\end{array}\right], \quad X(0)=\left[\begin{array}{ll}
1 & 0 \\
0 & 1
\end{array}\right] .
$$

Here, we use the Cauchy formula to obtain the solution of the system (23)

$$
\begin{aligned}
z(t) & =X(t) z_{0}+\int_{0}^{t} X(t-s)(u(s)-v(s)) d s \\
& =X(t)\left(z_{0}+\int_{0}^{t} X(-s)(u(s)-v(s))\right) d s=X(t) Y(t)
\end{aligned}
$$

Now $z(t)=0$ is equivalent to $Y(t)=0$. Therefore the differential game described by (22) is equivalent to that described by

$$
\dot{Y}(t)=X(-t)(u(t)-v(t)), \quad Y(0)=z_{0} .
$$

This system has the form (4), and so Theorem 7 applies. 


\section{Conclusion}

We have examined a linear pursuit-evasion differential game. We constructed a pair of optimal strategies for the players and gave formulae to find the optimal pursuit time.

That the game (1-2) can be completed for the first absorption time $\theta$ defined by (6) is familiar [16]. The important point of our investigation is the construction of the strategy of the Evader based on the position information; moreover, it is in an explicit form. As $z_{0} \neq 0$, then Lemma 10 can be reformulated as follows

If $\rho \leqslant \sigma$, then for any $\mathrm{T}>0$ evasion is possible on $[0, \mathrm{~T}]$ from $z_{0}$.

We stress that although by definition the Evader's strategy depends on $z$, only $z_{1}\left(t_{*}\right)$ is used in the construction of the strategy $V_{0}$.

Acknowledgements This research was partially supported by the National Fundamental Research Grant Scheme of Malaysia, No. 05-10-07-376FR.

\section{References}

[1] R. Isaacs. Differential games. John Wiley and Sons, New York, 1965. E60

[2] L. S. Pontryagin. Collected works. Nauka, Moscow, 1988. (Russian) E60

[3] L. D. Berkovitz. Necessary conditions for optimal strategies in a class of differential games and control problems. SIAM Journal on Control, $\mathbf{5}$, 1-24, 1967. E60 
[4] L. D. Berkovitz. A survey of differential games. Mathematical Theory of Control, Edited by A. V. Balakrishnan and L. W. Neustadt, Academic Press, New York, 373-385, 1967. E60

[5] N. N. Krasovskii and A. I. Subbotin. Game-theoretical control problems. New York, Springer, 1988. E60

[6] W. H. Fleming. The convergence problem for differential games. Journal of Mathematical Analysis and Applications. 3, 102-116, 1961. E60

[7] W. H. Fleming. The convergence problem for differential games, Part 2. Advances in Game Theory, Annals of Mathematics Studies, (52), Princeton University Press, Princeton, New Jersey,195-210, 1964. E60

[8] A. Friedman. Differential games. Wiley-Interscience, New York, 1971. E60

[9] R. J. Elliott and N. J. Kalton. The existence of value in differential games. Memoirs of the American Mathematical Society, 126, 1-67, 1972. E60

[10] L. A. Petrosyan. Differential games of pursuit. World Scientific, Singapore, London, 1993. E60

[11] O. Hajek. Pursuit games. Academic Press, New York, San Francisco, 1975. E60

[12] A. Ya. Azimov. Linear differential pursuit game with integral constraints on the control. Differentsial'nye Uravneniya, 11(10), 1975, 1723-1731; English transl. in Differential Equations 11, 1283-1289, 1975. E60

[13] A. Ya. Azimov. A linear differential evasion game with integral constraints on the controls. USSR Computational Mathematics and Mathematical Physics, 14 (6), 56-65, 1974. E60

[14] M. S. Nikolskii. The direct method in linear differential games with integral constraints. Controlled systems, IM, IK, SO AN SSSR, (2), 49-59, 1969. E60 
[15] A. I. Subbotin and V. N. Ushakov. Alternative for an encounter-evasion differential game with integral constraints on the players controls. PMM 39(3), 387-396, 1975. E60

[16] V. N. Ushakov. Extremal strategies in differential games with integral constraints. PMM, 36(1), 15-23, 1972. E60, E61, E73

[17] B. N. Pshenichnii and Yu. N. Onopchuk. Linear differential games with integral constraints. Izvestige Akademii Nauk SSSR, Tekhnicheskaya Kibernetika, (1), 13-22, 1968. E60, E61

[18] A. A. Azamov, B. Samatov. $\pi$-strategy. An elementary introduction to the theory of differential games. National University of Uzbekistan. Tashkent, Uzbekistan, 2000. E61

[19] G. I. Ibragimov. A game problem on a closed convex set. Siberian Advances in Mathematics. 12(3), 16-31, 2002. E61

[20] G. I. Ibragimov. A problem of optimal pursuit in systems with distributed parameters. J. Appl. Math. Mech, 66(5), 719-724, 2003. E61

[21] E. B. Lee and and L. Markus. Foundations of optimal control theory, John Wiley \& Sons Inc., New York, 1967. E67

\section{Author addresses}

1. G. I. Ibragimov, INSPEM \& Dept. Maths, Universiti Putra Malaysia, MALAYSIA

2. A. A. Azamov, Institute of Mathematics, UzBeKISTAN

3. M. Khakestari, Institute for Mathematical Research, Universiti Putra Malaysia, Malaysia

mailto:marzieh@inspem. upm . edu . my 